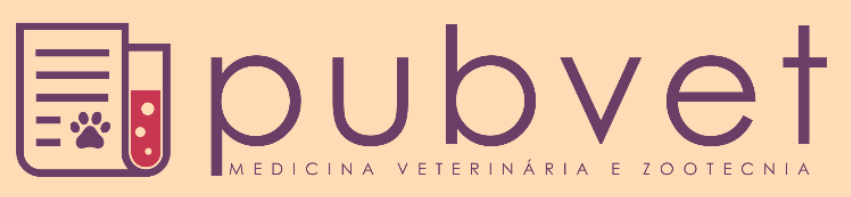

https://doi.org/10.31533/pubvet.v14n2a513.1-7

\title{
Estratégias para alterar a composição do leite caprino com reflexos para a saúde humana
}

\author{
Nycolle Cristyane Martins Freitas ${ }^{1 \bullet}$, Emília Maricato Pedro dos Santos ${ }^{2 *} \bullet$ \\ ${ }^{I}$ Graduanda em Medicina Veterinária da Universidade Federal de Juiz de Fora, Faculdade de Medicina, Departamento de Medicina \\ Veterinária. Juiz de Fora -MG Brasil. \\ ${ }^{2}$ Professora da Universidade Federal de Juiz de Fora, Faculdade de Medicina, Departamento de Medicina Veterinária. Juiz de Fora-MG Brasil. \\ *Autor para correspondência, E-mail: emilia.maricato@ufjf.edu.br
}

Resumo. A cabra doméstica (Capra aegagrushircus) foi um dos primeiros ruminantes domesticados pelo homem e possui características vantajosas, tais como crescimento rápido, alta progênie, alta adaptabilidade e resistência a condições climáticas e ambientais extremas. A procura do leite de cabra vem crescendo nos últimos tempos por se apresentar como uma alternativa bastante nutritiva para o ser humano sobretudo pela presença de componentes bioativos nesse alimento. O objetivo desta revisão literária é abordar sobre essa atividade biológica vantajosa para a alimentação humana mencionando inclusive estratégias utilizadas para influenciar na concentração dos compostos bioativos presentes no leite caprino. É possível verificar que o leite de cabra é capaz de modular processos metabólicos e levar a alguns efeitos benéficos para a saúde humana, como regulação do sistema imunológico, ação antioxidante, atividade antibacteriana, prevenção de lesões musculares em atletas de alto rendimento, melhorar a digestibilidade, efeito antiaterogênico e propriedades anticarcinogênicas. A presença destes compostos classifica o leite de cabra como um alimento funcional, o qual pode sofrer alterações em sua composição principalmente pela dieta do animal, mas também por alterações na temperatura e clima no local de produção, e com o sistema de produção. Dessa forma, é possível inferir que o leite de cabra vem se mostrando uma alternativa às necessidades nutricionais humanas devido à presença em sua composição natural de compostos bioativos.

Palavras chave: alimento funcional, compostos bioativos, leite de cabra, saúde humana

\section{Strategies to change goat milk composition with consequences for human health}

Abstract. The domestic goat (Capra aegagrushircus) was one of the first mandomesticates ruminants and presents great benefits such as rapid development, high progeny, high adaptability and resistance to extreme weather and environmental conditions. Lately, the demand for goat milk has been increasing as it presents itself as a very nutritious alternative for humans, especially due to the presence of bioactive components. This literature review aims at remark this beneficial biological activity for human health, including strategies to induce higher concentration of bioactive components in goat milk. This product is able to modulate metabolic processes and induce some beneficial effects on human health, such as immunomodulatory activity, antioxidant, antibacterial properties, prevention of injuries in high performance athletes, improving digestibility, antiatherogenic effect and anticarcinogenic properties. The presence of these compounds classifies goat milk as a functional food, and its composition can change mainly due to the animal's diet, but also by changes in weather conditions and within the farming 
system. Then, goat milk really has been an alternative to human nutritional requirements due to the presence of bioactive components in its composition.

Keywords: functional food, bioactive componentes, goat milk, human health

\section{Estrategias para cambiar la composición de la leche de cabra con consecuencias a la salud humana}

Resumen. La cabra doméstica (Capra aegagrushircus) fue uno de los primeros rumiantes domesticados por el hombre y presenta grandes ventajas como un rápido desarrollo, alta progenie, alta adaptabilidad y resistencia a condiciones climáticas y ambientales extremas. Últimamente, la demanda de leche de cabra ha aumentado, ya que se presenta como una alternativa muy nutritiva para los humanos, especialmente debido a la presencia de componentes bioactivos. Así que esta revisión de literatura tiene como objectivo destacar esta actividad biológica favorable a la alimentación humana, incluidas las estratégias para inducir una mayor concentración de componentes bioactivos en la leche de cabra. Este produto es capaz de modular los procesos metabólicos e inducir algunos efectos beneficiosos a la salud humana, como la actividad inmumoduladora, las propiedades antioxidantes, antibacterianas, la prevención de lesiones em atletas de alto rendimiento, la mejora de digestibilidad, el efecto antiaterogénico y las propiedades anticancerígenas. La presencia de estos compuestos clasifica la leche de cabra como um alimento funcional, y su composición puede cambiar principalmente debido a la dieta del animal, pero también a los câmbios en las condiciones climáticas y aún al sistema agrícola. Por lo tanto, la leche de cabra realmente ha sido una alternativa a los requerimientos nutricionales humanos debido a la presencia de componentes bioactivos en su composición.

Palavras clave: Alimento funcional, componentes bioactivos, leche de cabra, salud humana

\section{Introdução}

A cabra doméstica (Capra aegagrushircus) é uma espécie de pequeno ruminante amplamente distribuída que é mantida principalmente para carne, leite, couro e pelo. Esses animais são criados em uma ampla gama de sistemas de produção em todo o mundo, sendo a maioria criada em pequenos sistemas agrícolas mistos.

Os caprinos apresentam características biológicas e economicamente positivas, como crescimento rápido, alta progênie, alta adaptabilidade e resistência a condições climáticas e ambientais extremas (Jainudeen et al., 2004). Foram uns dos primeiros ruminantes domesticados pelos homens, cerca de 10.000 anos atrás, originários na região do atual Iraque, Irã, Síria e leste da Turquia (Zeuner, 1963).

Boer é a raça de carne mais popular, enquanto Angora e Cashmere são as principais raças produtoras de fibras. Algumas raças leiteiras, como Saanen, Toggenburg, Alpine e Anglo-Nubian, são comuns e frequentemente encontradas em rebanhos comerciais em todo o mundo (Ahuya et al., 2009; Ferreira et al., 2014; Medeiros et al., 2004; Simplício et al., 2004; Tholon et al., 2001).

De acordo com a FAO (2017), em 2016, havia quase 1 bilhão de caprinos em todo o mundo. Nos últimos 50 anos, a sua população multiplicou-se 2,4 vezes, enquanto outras espécies mantiveram ou diminuíram suas populações, com as tendências do mercado sugerindo que em 2030 o rebanho mundial caprino aumentará em mais de 50\% (Pulina et al., 2018).

A produção mundial de leite de cabra aumentou a uma taxa semelhante. Sua procura vem crescendo por ser de fácil digestão e rico em vitaminas, minerais e aminoácidos essenciais, tornando-se uma valiosa fonte de nutrientes. É recomendado para crianças, idosos e pessoas em recuperação de doenças ou distúrbios digestivos (Kukovics, 2016) e novos conhecimentos agregados à atividade biológica deste leite vêm sendo publicados (García et al., 2014).

O objetivo deste trabalho é abordar a atividade biológica do leite de cabra vantajosa para a alimentação humana bem como estratégias utilizadas para influenciar na concentração dos compostos bioativos presentes no leite caprino. 


\section{Características do leite caprino}

A composição do leite de cabra pode variar de acordo com fatores genéticos, fisiológicos, ambientais e de manejo (Verruck et al., 2019). Embora os teores de sólidos totais (ST), gordura, proteína, lactose e cinzas do leite de cabra sejam semelhantes ao bovino, diferenças importantes são encontradas entre os componentes individuais de ambos os tipos de leite (Park et al., 2007).

Deve-se ter atenção à saúde e bem-estar do animal para manter a produção de leite adequada, associados a isto, os efeitos ambientais podem desencadear alterações comportamentais e fisiológicas, que resultam em baixos índices produtivos (Borburema et al., 2013).

García et al. (2014) afirmam que um dos fatores decisivos no crescimento do consumo de leite de cabra e derivados é a percepção de efeitos benéficos para a saúde humana, que, além disso, são plenamente reconhecidos pela comunidade científica. Dentre esses efeitos, o leite de cabra tem um odor e sabor atraentes e aceitáveis e pode ser consumido como uma alternativa ao leite porque é menos alergênico (Park et al., 2007), possui melhor digestibilidade (García et al., 2014) e valores terapêuticos na pediatria, na gastrenterologia e na nutrição humana (Amaral et al., 2011; Amigo \& Fontecha, 2011).

\section{Leite caprino como alimento funcional}

A convicção de desenvolver alimentos funcionais surgiu pela primeira vez no Japão na década de 1980, quando aquele país se deparou com custos crescentes de cuidados de saúde. A princípio o Ministério da Saúde e Bem-Estar japonês criou um sistema regulador para aprovar certos alimentos documentados como benéficos para saúde (Arai, 1996). O termo "alimento funcional" apareceu pela primeira vez em 1993 na revista Nature, sob o título "O Japão explora a fronteira entre alimentos e remédios" (Swinbanks \& O'Brien, 1993). Existem algumas definições para alimento funcional por não ser um termo reconhecido em lei, mas sim por ser um termo de marketing. A Health Canada (2000) define como "similar na aparência aos alimentos convencionais, consumidos como parte da dieta habitual, com benefícios fisiológicos demonstrados e/ou para reduzir o risco de doenças crônicas além das funções nutricionais básicas". No Japão esses alimentos são reconhecidos como uma categoria distinta e como alimentos para uso específico da saúde, sendo intitulados como Food for Specified Health Uses (FOSHU) e definidos como "alimentos compostos por ingredientes funcionais que afetam a estrutura e/ou função do corpo e são utilizados para manter ou regular condições específicas de saúde, como saúde gastrointestinal, pressão arterial e níveis de colesterol sanguíneo" (Hosoya, 1998). Anos depois, o conceito de alimentos funcionais foi ampliado para incluir componentes alimentares que reduzem o risco de doenças crônicas (Plat \& Mensink, 2001).

Henry (2010) agrupa os alimentos funcionais como:

- Alimentos convencionais contendo substâncias bioativas naturais;

- $\quad$ Alimentos que foram modificados, por enriquecimento ou outros meios, com substâncias bioativas;

- Ingredientes alimentares sintetizados, como alguns carboidratos especializados, destinados a ter efeitos probióticos.

Atualmente sabe-se que o leite de cabra pode ser digerido facilmente, é rico em minerais e aminoácidos essenciais, e tem um valor nutricional muito alto. Os laticínios caprinos agora têm um lugar notável na nutrição saudável moderna e podem ser considerados alimentos funcionais, especialmente quando os animais são criados em condições orgânicas, enquadrando-se no tipo alimentos convencionais contendo substâncias bioativas naturais (Kukovics, 2016).

\section{Compostos bioativos presentes no leite caprino}

De acordo com Galanakis (2016), compostos bioativos são componentes presentes no alimento funcional, essenciais ou não essenciais, capazes de afetar o corpo modulando processos metabólicos. A eles são relacionados alguns efeitos benéficos como atividade antioxidante, estimulante de mecanismos de defesa, inibição ou indução de enzimas e de expressão gênica (Correia et al., 2012). 
Dentre esses compostos bioativos, as proteínas do leite de cabra têm ganhado cada vez mais atenção, especialmente os peptídeos bioativos formados das proteínas parentais pelas enzimas digestivas. No estudo feito por Ahmed et al. (2015), para atividades de remoção de radicais livres, foram examinados peptídeos gerados a partir da hidrólise por pepsina de caseínas e proteínas de soro de leite de cabra. Os resultados demonstraram uma notável capacidade destes peptídeos de eliminar os radicais superóxidos e uma alternativa interessante para o uso de resíduos (soro de leite) da indústria de queijo. Este estudo ainda relata que hidrolisados de pepsina e / ou peptídeos isolados de frações de proteína caprina são uma oportunidade de candidatura terapêutica na indústria farmacêutica para tratamento ou prevenção do estresse oxidativo e suas doenças associadas. Um número consistente de evidências sugere associação do estresse oxidativo a condições clínicas como doenças cardiovasculares, diabetes mellitus, neoplasias, doenças respiratórias e distúrbios neurológicos (Gottlieb et al., 2010). Em estudos feitos em camundongos, utilizando leite de cabra fermentado, o teste de hipersensibilidade do tipo retardado (DTH) realizado sete semanas após a imunização primária nos camundongos que consumiram leite de cabra fermentado mostrou uma reação DTH mais elevada do que nos camundongos controle. Houve também um aumento do tempo de natação no teste de natação. Estes resultados sugerem que a administração de leite de cabra fermentado aumenta não apenas as respostas imunes contra antígenos, mas também a força física (Oh et al., 2016; Ribeiro \& Ribeiro, 2010).

As beta-lactamases de espectro estendido (ESBLs) são enzimas produzidas por bactérias patogênicas que permitem que esses micro-organismos sejam resistentes a múltiplas drogas. As ESBLs produzidas pela Escherichia coli são resistentes às seguintes bases farmacológicas antimicrobianas: cefazolina $(96,6 \%)$, ceftazidima $(43,3 \%)$, ceftriaxona $(3,3 \%)$, ciprofloxacina $(76,6 \%)$, meropenem $(0 \%)$, tobramicina $(43,3 \%)$, cefotaxima $(93,3 \%)$, ceftazidima + ido cluvalico $(3,3 \%)$, cefotaxima + ido cluvalico $(3,3 \%)$, cefperodoxima $(96,6 \%)$, piperacilina + tazobactam $(0 \%)$, ampicilina $(100 \%)$. As espécies de Lactobacillus isoladas de leite de cabra fermentado exibiram um efeito antibacteriano em uma faixa estreita de cepas ESBL. No entanto, o efeito in vitro é melhor do que o grande número de antibióticos de amplo espectro tais como as cefalosporinas de terceira geração (

O ácido siálico tem se mostrado em estudos benefícios à saúde humana, dentre eles: promover o desenvolvimento do cérebro infantil referindo-se a parte de cognição e memória, atuar na regulação do sistema imunológico e na função intestinal agindo contra enterotoxinas e infecções nos recém-nascidos, entre outros efeitos fisiológicos importantes (Hernández-Ledesma et al., 2011). No estudo apresentado por Pereira (2016) demonstrou-se que a coagulação enzimática do soro de queijos caprinos preserva o ácido siálico (Neu5Gc e Neu5Ac) mais eficientemente do que a coagulação mista e mostra que, devido à quantidade desse importante nutriente encontrado, o soro de queijo caprino é uma fonte potencial de compostos bioativos com efeitos benéficos para a saúde humana e uma alternativa econômica e ecologicamente viável para a indústria de alimentos inserir em produtos destinados à alimentação de crianças.

A taurina é particularmente rica no leite de cabra, sendo 20 a 40 vezes maior do que o leite de origem bovina (Mehaia \& Al-Kanhal, 1992). De acordo com Silanikove et al. (2010), a taurina está envolvida na formação de sais biliares, osmorregulação, antio-xidação, transporte de cálcio e no sistema nervoso central. Carvalho (2015) afirma que a suplementação de taurina pode ser considerada como um possível recurso ergogênico com a finalidade de prevenir o catabolismo proteico e favorecer a manutenção da massa magra, além de reduzir danos oxidativos decorrentes da atividade física intensa, e, desta forma, pode prevenir lesões musculares e beneficiar o desempenho de triatletas.

Amaral et al. (2011) afirma que a melhor digestibilidade do leite de cabra em relação ao leite bovino ocorre devido a fatores como o alto conteúdo de ácidos graxos de cadeia curta (compostos de 4 a 10 átomos de carbono). Os glóbulos de gordura no leite caprino são menores em tamanho, promovendo maior área superficial para degradação enzimática, o que confere maior eficiência na absorção e digestão pelo organismo humano (Silva et al., 2015).

Vale ressaltar também a presença de elevadas proporções de ácidos graxos poli-insaturados (ômega3 e ômega-6), com propriedades relacionadas a condições cardiovasculares, bem como ácido linoleico conjugado (CLA), nutriente de efeito antiaterogênico e propriedades anticarcinogênicas, conforme afirmado por Slačanac et al. (2010) e ação sobre a função cardiovascular, na redução de gordura e peso corporal (Rodrigues et al., 2014). 


\section{Estratégias para alterar a composição do leite caprino}

A composição da dieta do animal tem sido a maior evidência de que é possível afetar a concentração de componentes bioativos em leite de ruminantes; porém, pode ser afetada também por alterações na temperatura e clima no local de produção, assim como pelo sistema de produção (Batista et al., 2017). Jaafar et al. (2018) concluíram que além da variação genética entre as raças de caprinos, a alimentação desses animais composta por alto teor de lipídios e fibras, como, por exemplo, com produtos à base de soja, pode levar ao aumento do teor de gordura no leite de cabra.

Em pesquisa realizada por Gómez-Cortés et al. (2019) o uso de óleo de linhaça na dieta de cabras demonstrou o aumento significativo, entre 12 e 24 h após sua inclusão na dieta, do ácido graxo ômega3 e do ácido linoleico conjugado, bem como diminui a saturação de ácido graxo na gordura do leite, o que melhora o valor nutricional dos produtos lácteos de cabra.

Boutoial et al. (2013) concluíram em seu estudo que a introdução de Rosmarinus officinalis spp. (alecrim) em 20\% na dieta das cabras aumentou o teor de ácidos graxos poli-insaturados e permitiu uma adequação tecnológica do leite, reduzindo o tempo de coagulação do mesmo no processo de fabricação de queijos. Com isso, a suplementação de alecrim a $20 \%$ parece ser uma alternativa alimentar para cabras adequada para a produção de leite e queijos com benefícios para a saúde humana, e, ainda, sem alterações nas suas propriedades sensoriais.

\section{Considerações finais}

$\mathrm{O}$ leite de cabra vem se mostrando uma alternativa às necessidades nutricionais humanas devido à presença de componentes bioativos. Levando-se em conta que os efeitos benéficos são reconhecidos pela comunidade científica, seu estudo mais aprofundado é interessante para a valorização tanto do produto na forma natural, quanto na sua aplicação pela indústria de alimentos.

\section{Referências bibliográficas}

Ahmed, A. S., El-Bassiony, T., Elmalt, L. M. \& Ibrahim, H. R. (2015). Identification of potent antioxidant bioactive peptides from goat milk proteins. Food Research International, 7480-88.

Ahuya, C. O., Ojango, J. M. K., Mosi, R. O., Peacock, C. P. \& Okeyo, A. M. (2009). Performance of Toggenburg dairy goats in smallholder production systems of the eastern highlands of Kenya. Small Ruminant Research, 83(1-3):7-13.

Amaral, R. M. 1., Macedo, F. F. A., Alcalde, C. R., Lino, D. A., Bánkuti, F. I., Macedo, F. G., .. Gualda, T. P. (2011). Desempenho produtivo e econômico de cordeiros confinados abatidos com três espessuras de gordura. Revista Brasileira de Saúde e Produção Animal, 12(1):155-165.

Amigo, L. \& Fontecha, J. (2011). Milk | Goat Milk. In J. W. Fuquay (Ed.), Encyclopedia of Dairy Sciences (Second Edition) (pp. 484-493). San Diego: Academic Press.

Arai, S. (1996). Studies on functional foods in Japan—state of the art. Bioscience, Biotechnology, and Biochemistry, 60(1):9-15.

Batista, A. S. M., Sousa, Y. R. F. \& Queiroga, R. C. R. E. (2017). Compostos bioativos para agregação de valor dos derivados do leite caprino. Revista Científica de Produção Animal, 19(1):26-35.

Borburema, J. B., Souza, B. B., Cezar, M. F. \& Pereira Filho, J. M. (2013). Influência de fatores ambientais sobre a produção e composição físico-química do leite. Agropecuária Científica no Semiárido, 9(4):15-19.

Boutoial, K., Ferrandini, E., Rovira, S., García, V. \& López, M. B. (2013). Effect of feeding goats with rosemary (Rosmarinus officinalis spp.) by-product on milk and cheese properties. Small Ruminant Research, 112(1-3):147-153.

Carvalho, F. G. (2015). Efeitos da suplementação de taurina ou flavonóides de cacau associado à achocolatado sobre a composição corporal, metabolismo de carboidratos e proteinas, desempenho fisico, dano muscular e estresse oxidativo em atletas. PhD, Universidade Estadual Paulista, Araraquara, São Paulo, Brasil. 
Correia, R. T. P., Borges, K. C., Medeiros, M. F. \& Genovese, M. I. (2012). Bioactive compounds and phenolic-linked functionality of powdered tropical fruit residues. Food Science and Technology International, 18(6):539-547.

FAO. (2017). Statistical Yearbook (Vol. 1). Rome, Italy: Food and Agriculture Organization of the United Nations.

Ferreira, T. A., Pereira, I. G., Gouveia, A. M. G., Pires, A. V., Facó, O., Farah, M. M., . . Guimarães, M. (2014). Avaliação genética de caprinos da raça Saanen nascidos no Brasil de 1979 a 2009. Arquivo Brasileiro de Medicina Veterinária e Zootecnia, 661179-1188.

Galanakis, C. M. (2016). Nutraceutical and functional food components: effects of innovative processing techniques. Massachusetts, USA.: Academic Press.

García, V., Rovira, S., Boutoial, K. \& López, M. B. (2014). Improvements in goat milk quality: A review. Small Ruminant Research, 121(1):51-57.

Gómez-Cortés, P., Cívico, A., de la Fuente, M. A., Sánchez, N. N., Blanco, F. P. \& Marín, A. L. M. (2019). Short term evolution of nutritionally relevant milk fatty acids of goats fed a cereal-based concentrate enriched with linseed oil. Innovative Food Science \& Emerging Technologies, 51107113.

Gottlieb, M. G. V., Cruz, I. B. M., Schwanke, C. H. A. \& Bodanese, L. C. (2010). Estresse oxidativo como fator de risco cardiometabólico emergente. Scientia Medica, 20(3):243-249.

Henry, C. J. (2010). Functional foods. European Journal of Clinical Nutrition, 64(7):657-659.

Health Canada. (2000). Standards of evidence for evaluating foods with health claims: a proposed framework consultation document. June.

Hernández-Ledesma, B., Ramos, M. \& Gómez-Ruiz, J. Á. (2011). Bioactive components of ovine and caprine cheese whey. Small Ruminant Research, 101(1-3):196-204.

Hosoya, N. (1998). Health claims in Japan, foods for specified health use and functional foods. Jornal of Nutrition Food, 11-11.

Jaafar, S. H. S., Hashim, R., Hassan, Z. \& Arifin, N. (2018). A comparative study on physicochemical characteristics of raw goat milk collected from different farms in Malaysia. Tropical Life Sciences Research, 29(1):195-212.

Jainudeen, M., Wahid, H. \& Hafez, E. (2004). Ovinos e caprinos. In E. S. E. Hafez \& B. Hafez (Eds.), Reprodução Animal (pp. 172-182). São Paulo: Manole.

Kukovics, S. (2016). Sustainable goat breeding and goat farming in the Central and Eastern European countries. Paper presented at the Sustainable Goat Breeding and Goat Farming in the Central and Eastern European Countries Debrecen (Hungary).

Medeiros, L. F. D., Luna, M. C. M. d., Cabral Neto, O. \& Vieira, D. H. (2004). Avaliação de alguns aspectos de desempenho de caprinos da raça Anglo-nubiana, no Estado do Rio de Janeiro. Revista de Ciências da Vida, 24103-118.

Mehaia, M. A. \& Al-Kanhal, M. A. (1992). Taurine and other free amino acids in milk of camel, goat, cow and man. Milchwissenschaft, 47(6):351-353.

Oh, K.-H., Kim, A. R., Bae, J.-H., Lee, K. B. \& Yoo, Y. C. (2016). Effects of fermented Goat milk on immunomodulatory activity and physical strength in mice. Journal of the Korean Society of Food Science and Nutrition, 45(2):174-180.

Park, Y. W., Juárez, M., Ramos, M. \& Haenlein, G. F. W. (2007). Physico-chemical characteristics of goat and sheep milk. Small Ruminant Research, 68(1-2):88-113.

Pereira, N. L. V. (2016). Concentração de àcido siálico em soro de queijo caprino resultante do processo de coagulação láctea enzimática e mista. Master of Science, Universidade Federal de Pernambuco, Recife, Pernambuco, Brasil.

Plat, J. \& Mensink, R. P. (2001). Effects of plant sterols and stanols on lipid metabolism and cardiovascular risk. Nutrition, Metabolism, and Cardiovascular Diseases, 11(1):31-40. 
Pulina, G., Milán, M. J., Lavín, M. P., Theodoridis, A., Morin, E., Capote, J., . . Caja, G. (2018). Invited review: Current production trends, farm structures, and economics of the dairy sheep and goat sectors. Journal of Dairy Science, 101(8):6715-6729.

Ribeiro, A. C. \& Ribeiro, S. D. A. (2010). Specialty products made from goat milk. Small Ruminant Research, 89(2-3):225-233.

Rodrigues, R., Soares, J., Garcia, H., Nascimento, C., Medeiros, M., Bomfim, M., . . Queiroga, R. (2014). Goat milk fat naturally enriched with conjugated linoleic acid increased lipoproteins and reduced triacylglycerol in rats. Molecules, 19(3):3820-3831.

Silanikove, N., Leitner, G., Merin, U. \& Prosser, C. G. (2010). Recent advances in exploiting goat's milk: Quality, safety and production aspects. Small Ruminant Research, 89(2-3):110-124.

Silva, G. J., Gonçalves, B.-H. R. F., Conceição, D. G., Pontes, S. F. O. \& Ferrão, S. P. B. (2015). Perfil de ácidos graxos e frações proteicas do leite de cabra. Revista do Instituto de Laticínios Cândido Tostes, 70(6):338-348.

Simplício, A. A., Wander, A. E., Leite, E. R. \& Lopes, E. A. (2004). A caprino-ovinocultura de corte como alternativa para a geração de emprego e renda. Paper presented at the Embrapa Caprinos. Documentos, Mossoró.

Slačanac, V., Božanić, R., Hardi, J., Rezessyné, S. J., Lučan, M. \& Krstanović, V. (2010). Nutritional and therapeutic value of fermented caprine milk. International Journal of Dairy Technology, 63(2):171-189.

Suresh, P., Reddy, V. S. \& Vamsimunikrishna, P. (2018). Antibacterial activity of Lactobacillus (LB) strains isolated from goat milk against ESBL producing E. coli causing wound infections. Indian Journal of Public Health Research \& Development, 9(7):281-285.

Swinbanks, D. \& O'Brien, J. (1993). Japan explores the boundary between food and medicine. Nature, 364(6434):180-180.

Tholon, P., Queiroz, S. A., Ribeiro, A. C., Resende, K. T. \& Ribeiro, S. D. A. (2001). Estudo genético quantitativo da produção de leite em caprinos da raça Saanen. Archivos Latinoamericanos Producción Animal, 9(1):1-5.

Verruck, S., Dantas, A. \& Prudencio, E. S. (2019). Functionality of the components from goat's milk, recent advances for functional dairy products development and its implications on human health. Journal of Functional Foods, 52243-257.

Zeuner, F. E. (1963). A history of domesticated animals. Harper \& Row Publishers, New York, New York, USA. New York, USA.: Harper \& Row Publishers.

Recebido: 23 de setembro, 2019.

Aprovado: 10 de outubro, 2019.

Publicado: 21 de março, 2020.

Licenciamento: Este artigo é publicado na modalidade Acesso Aberto sob a licença Creative Commons Atribuição 4.0 (CC-BY 4.0), a qual permite uso irrestrito, distribuição, reprodução em qualquer meio, desde que o autor e a fonte sejam devidamente creditados 